\title{
A Site-Selectivity Switch in Lewis Acid Catalysis. Mechanism and Kinetic Simulation of Skeletal Rearrangement of Cyclobutene-fused Homoquinones
}

Ken Kokubo, Takuya Koizumi, Kenji Harada, Eiko Mochizuki, and Takumi Oshima*

Division of Applied Chemistry, Graduate School of Engineering, Osaka University,

Suita, Osaka 565-0871, Japan

\section{Supporting Information}

Table of Contents

General experimental information $\quad$ S2

Table of product distribution (Table S1, S2) S3

$\begin{array}{ll}\text { Compound data } & \text { S4 }\end{array}$

X-ray crystallographic data with ORTEP S6

$\begin{array}{ll}\text { Calculation information } & \text { S8 }\end{array}$

$\begin{array}{ll}\text { References } & \text { S8 }\end{array}$ 


\section{Experimental}

Materials. Cyclobutene-fused homobenzoquinones $\mathbf{1 a}, \mathbf{b}$ were synthesized by the [2+2] photocycloaddition of the corresponding homobenzoquinone with alkynes as previously described. ${ }^{\mathrm{S} 1}$ The rearrangement products were isolated by HPLC equipped with a semifractionation ODS column and recrystallized from hexane-benzene. The compounds 1a, 2a, $4 \mathbf{a}$ and $\mathbf{5 a}$ were already reported (3a was not formed). ${ }^{\mathrm{S} 2}$ The new compounds $\mathbf{1 b}, \mathbf{2 b}, \mathbf{3 b}$ and $\mathbf{5 b}$ were identified by ${ }^{1} \mathrm{H}$ and ${ }^{13} \mathrm{C}$ NMR as well as IR spectra as follows (4b was not formed), and also the structure of $\mathbf{3 b}$ and $\mathbf{5 b}$ were confirmed by X-ray crystallographic analysis.

\section{General Procedure for $\mathrm{BF}_{3} \cdot \mathbf{E t}_{2} \mathrm{O}$ Catalyzed Reactions of $\mathbf{1}$.}

$\mathrm{BF}_{3} \cdot \mathrm{Et}_{2} \mathrm{O}(7.6 \mu \mathrm{L}, 0.06 \mathrm{mmol})$ were added into a $\mathrm{CDCl}_{3}$ solution $(670 \mu \mathrm{L})$ of $\mathbf{1 a}(8.36 \mathrm{mg}, 0.02$ $\mathrm{mmol}$ ) in a NMR tube using a micro syringe at room temperature. The progress of reaction was monitored by ${ }^{1} \mathrm{H}$ NMR. After a period of requisite time, the reaction solution was transferred into a separate funnel, diluted with chloroform $(10 \mathrm{~mL})$ and then washed with water $(3 \mathrm{~mL} \times 3)$. The aqueous layer was extracted with chloroform $(5 \mathrm{~mL}$ x 2). The combined organic layer was washed with water $(3 \mathrm{~mL} \times 3)$, then dried over calcium chloride. After the evaporation of the solvent, the residue was submitted for a ${ }^{1} \mathrm{H}$ NMR analysis to determine the product distribution. 
Table S1. Product distribution on rearrangement of $1 \mathbf{a}$

\begin{tabular}{cccccccc}
\hline Equiv of $\mathrm{BF}_{3}$ & Time $/ \mathrm{h}$ & Conv $/ \%$ & $\mathbf{2 a}$ & $\mathbf{( 3 a )}$ & $\mathbf{4 a}$ & $\mathbf{5 a}$ & $\mathrm{P}_{\mathrm{A}}: \mathrm{P}_{\mathrm{B}}$ \\
\hline 0.5 & 120 & 67 & 69 & $8^{a}$ & 5 & 18 & $69: 31$ \\
1 & 70 & 100 & 66 & - & 7 & 27 & $66: 34$ \\
1.5 & 47 & 100 & 56 & - & 7 & 37 & $56: 44$ \\
2 & 8 & 100 & 54 & - & 4 & 42 & $54: 46$ \\
3 & 2 & 100 & 44.4 & - & - & 55.6 & $44.4: 55.6$ \\
10 & 1 & 100 & 32 & - & - & 68 & $32: 68$ \\
20 & 1 & 100 & 23 & - & - & 77 & $23: 77$ \\
50 & 3 & 100 & 8.3 & - & - & 91.7 & $8.3: 91.7$ \\
100 & 3 & 100 & 3.9 & - & - & 96.1 & $3.9: 96.1$ \\
264 & 0.5 & 100 & 3.0 & - & - & 97.0 & $3.0: 97.0$ \\
\hline
\end{tabular}

a Tentative.

Table S2. Product distribution on rearrangement of $\mathbf{1 b}$

\begin{tabular}{cccccccc}
\hline Equiv of $\mathrm{BF}_{3}$ & Time / $\mathrm{h}$ & Conv /\% & $\mathbf{2 b}$ & $\mathbf{3 b}$ & $\mathbf{( 4 b )}$ & $\mathbf{5 b}$ & $\mathrm{P}_{\mathrm{A}}: \mathrm{P}_{\mathrm{B}}$ \\
\hline 0.5 & 120 & 71.4 & 82.1 & 17.9 & - & - & $82.1: 17.9$ \\
1 & 23 & 100 & 75.8 & 24.2 & - & - & $75.8: 24.2$ \\
2 & 17 & 100 & 74.0 & 26.0 & - & - & $74.0: 26.0$ \\
3 & 10 & 100 & 70.1 & 29.9 & - & - & $70.1: 29.9$ \\
6 & 6 & 100 & 63.5 & 36.5 & - & - & $63.5: 36.5$ \\
10 & 3 & 100 & 54.7 & 45.3 & - & - & $54.7: 45.3$ \\
20 & 2 & 100 & 40.5 & 59.5 & - & - & $40.5: 59.5$ \\
50 & 12 & 100 & 16.0 & 34.0 & - & 50.0 & $16.0: 84.0$ \\
100 & 5 & 100 & 11.3 & 27.5 & - & 61.2 & $11.3: 88.7$ \\
264 & 12 & 100 & 5.0 & 22.0 & - & 73.0 & $5.0: 95.0$ \\
\hline
\end{tabular}




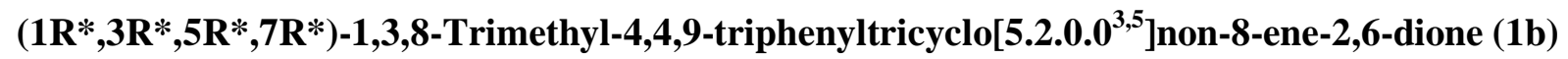

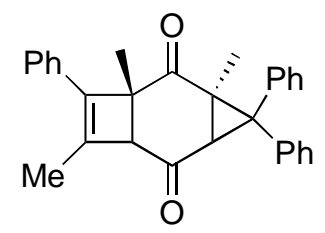

Mp. 162.2-163.0 ${ }^{\circ} \mathrm{C}$, white crystal; ${ }^{1} \mathrm{H}$ NMR $\left(270 \mathrm{MHz}, \mathrm{CDCl}_{3}\right) \delta 0.75(\mathrm{~s}, 3 \mathrm{H}), 1.16(\mathrm{~s}, 3 \mathrm{H}), 2.02(\mathrm{~d}$, $3 \mathrm{H}, J=1.6 \mathrm{~Hz}), 2.54(\mathrm{~d}, 1 \mathrm{H}, J=1.6 \mathrm{~Hz}), 2.73(\mathrm{~s}, 1 \mathrm{H}), 7.17-7.51(\mathrm{~m}, 15 \mathrm{H}) ;{ }^{13} \mathrm{C} \mathrm{NMR}(67.5 \mathrm{MHz}$, $\left.\mathrm{CDCl}_{3}\right) \delta 14.2,18.8,19.5,40.1,42.8,47.1,56.0,61.3,136.6,127.2,127.65,127.69,128.3,128.65$, 128.74, 128.9, 130.3, 132.6, 138.3, 138.4, 141.0, 143.7, 204.1, 208.1; IR (KBr) $1672(\mathrm{C}=\mathrm{O}) \mathrm{cm}^{-1}$; Anal. Calcd for $\mathrm{C}_{30} \mathrm{H}_{26} \mathrm{O}_{2}$ : C, 86.09; H, 6.26, Found: C, 86.28; H, 6.41.

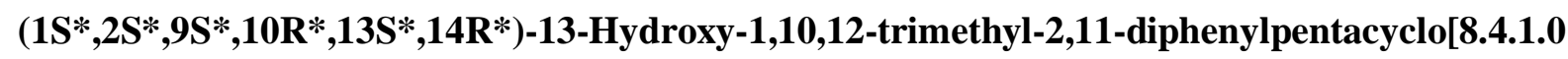
${ }^{2,14} .0^{3,8} .0^{9,13}$ pentadeca-3(8),4,6,11-tetraen-15-one (2b)

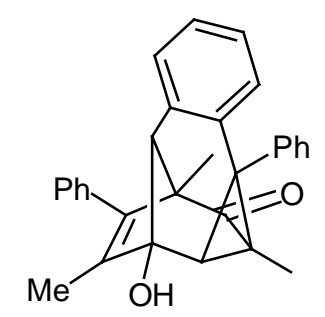

White crystal; ${ }^{1} \mathrm{H}$ NMR $\left(270 \mathrm{MHz}, \mathrm{CDCl}_{3}\right) \delta 1.03(\mathrm{~s}, 3 \mathrm{H}), 1.12(\mathrm{~s}, 3 \mathrm{H}), 1.93(\mathrm{~s}, 1 \mathrm{H}), 2.01(\mathrm{~s}, 3 \mathrm{H})$, $2.52(\mathrm{~d}, 1 \mathrm{H}, J=2.6 \mathrm{~Hz}), 3.53(\mathrm{~d}, 1 \mathrm{H}, J=2.6 \mathrm{~Hz}), 6.89(\mathrm{dd}, 1 \mathrm{H}, J=6.9,2.0 \mathrm{~Hz}), 7.06-7.52(\mathrm{~m}, 13 \mathrm{H})$; ${ }^{13} \mathrm{C} \mathrm{NMR}\left(67.5 \mathrm{MHz}, \mathrm{CDCl}_{3}\right) \delta 11.5,15.120 .0,38.9,43.6,48.1,63.1,68.6,75.9,126.0,127.1,127.5$, 127.6, 127.7, 128.0, 128.1, 128.3, 129.4, 130.0, 130.2, 131.3, 132.3, 135.3, 137.1, 138.3, 139.2, 147.2, 205.0 .

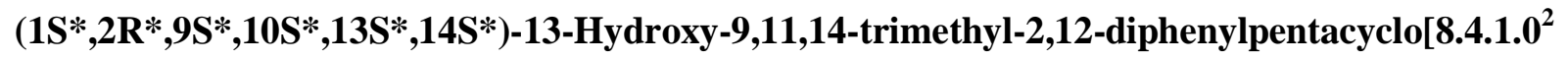
${ }^{, 14} .0^{3,8} .0^{9,13}$ ]pentadeca-3(8),4,6,11-tetraen-15-one (3b)

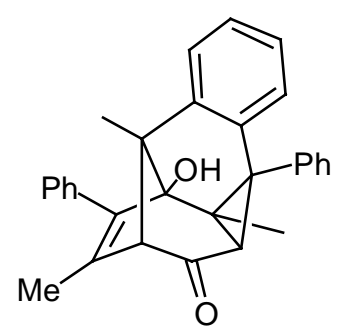

Mp. 285.5-286.5 ${ }^{\circ} \mathrm{C}$, white crystal; ${ }^{1} \mathrm{H}$ NMR $\left(270 \mathrm{MHz}, \mathrm{CDCl}_{3}\right) \delta 0.78(\mathrm{~s}, 3 \mathrm{H}), 1.80(\mathrm{~s}, 1 \mathrm{H}), 1.83(\mathrm{~s}$, $3 \mathrm{H}), 1.87(\mathrm{~s}, 3 \mathrm{H}), 2.47(\mathrm{~d}, 1 \mathrm{H}, J=1.0 \mathrm{~Hz}), 2.89(\mathrm{~s}, 1 \mathrm{H}), 6.56(\mathrm{dd}, 1 \mathrm{H}, J=1.0,8.2 \mathrm{~Hz}), 7.04-7.56(\mathrm{~m}$, $13 \mathrm{H}) ;{ }^{13} \mathrm{C} \mathrm{NMR}\left(67.5 \mathrm{MHz}, \mathrm{CDCl}_{3}\right) \delta 14.7,16.3,19.1,42.3,43.3,51.7,54.0,75.9,82.8,126.3,126.8$, $126.9,127.3,127.4,128.2,128.4,128.5,128.8,129.1,130.6,131.1,135.7,135.9,137.7,139.1,146.3$, 200.7; IR (KBr): 3480 (br, OH), $1668(\mathrm{C}=\mathrm{O}) \mathrm{cm}^{-1}$. 
$\left(2 S^{*}, 4 R^{*}, 5 R^{*}, 6 R^{*}, 10 R^{*}\right)-2,5,9-T r i m e t h y l-4,10-$ diphenyltetracyclo $\left[9.4 .0 .0^{2,6} .0^{5,10}\right]$ pentadeca-1(1 5),8,11,13-tetraene-3,7-dione (5b)

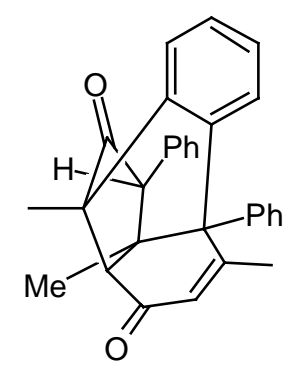

White crystal; ${ }^{1} \mathrm{H}$ NMR $\left(270 \mathrm{MHz}, \mathrm{CDCl}_{3}\right) \delta 1.66(\mathrm{~s}, 3 \mathrm{H}), 1.75(\mathrm{~s}, 3 \mathrm{H}), 1.80(\mathrm{~d}, 3 \mathrm{H}, J=1.0 \mathrm{~Hz}), 2.77$ $(\mathrm{s}, 1 \mathrm{H}), 3.65(\mathrm{~s}, 1 \mathrm{H}), 6.13(\mathrm{q}, 1 \mathrm{H}, J=1.0 \mathrm{~Hz}), 6.13-6.34(\mathrm{~m}, 4 \mathrm{H}), 6.88-7.46(\mathrm{~m}, 9 \mathrm{H}) ;{ }^{13} \mathrm{C} \mathrm{NMR}(67.5$ $\left.\mathrm{MHz}, \mathrm{CDCl}_{3}\right) \delta 17.0,21.9,27.2,49.9,56.9,59.0,63.4,66.2,125.4,125.5,125.9,126.7,127.1$, $127.90,127.93,128.4,128.69,129.74,129.9,130.7,132.6,132.8,134.2,134.8,138.2,168.9,194.1$, 206.7. 
X-ray Crystal structure Determination of 3b: $\mathrm{C}_{30} \mathrm{H}_{26} \mathrm{O}_{2}, \mathrm{M}=418.53$, monoclinic, space group $P 2{ }_{1} / c$ with $a=12.752(7), b=13.44(1), c=14.274(4) \AA, \beta=115.22(3)^{\circ}, V=2213.46 \AA^{3}, Z=4, D c=$ $1.256 \mathrm{~g} / \mathrm{cm}^{3}, R=0.079$ and $R w=0.079$ for 3380 reflections with $I>3.00 \sigma(I)$.

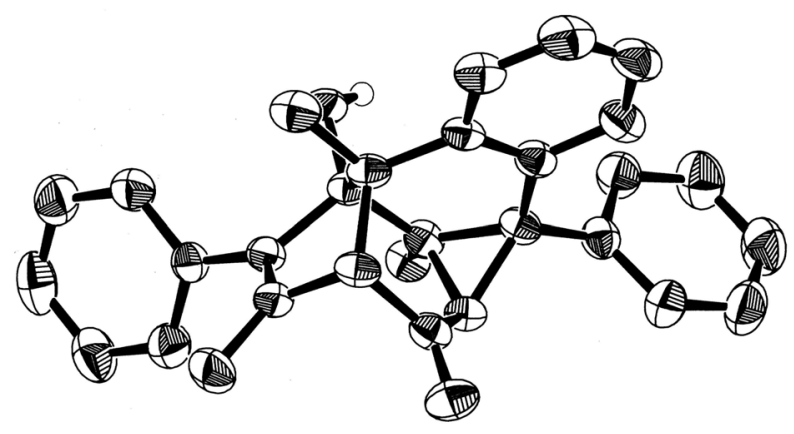

Figure S2. ORTEP drawing of $\mathbf{3 b}$.

X-ray Crystal structure Determination of $5 \mathbf{b}: \mathrm{C}_{30} \mathrm{H}_{26} \mathrm{O}_{2}, \mathrm{M}=418.53$, monoclinic, space group $P 2_{1} / c$ with $a=15.503(5), b=8.505(2), c=16.954(3) \AA, \beta=100.37(2)^{\circ}, V=2199.0(9) \AA^{3}, Z=4, D c$ $=1.264 \mathrm{~g} / \mathrm{cm}^{3}, R=0.070$ and $R w=0.054$ for 2657 reflections with $I>3.00 \sigma(I)$.

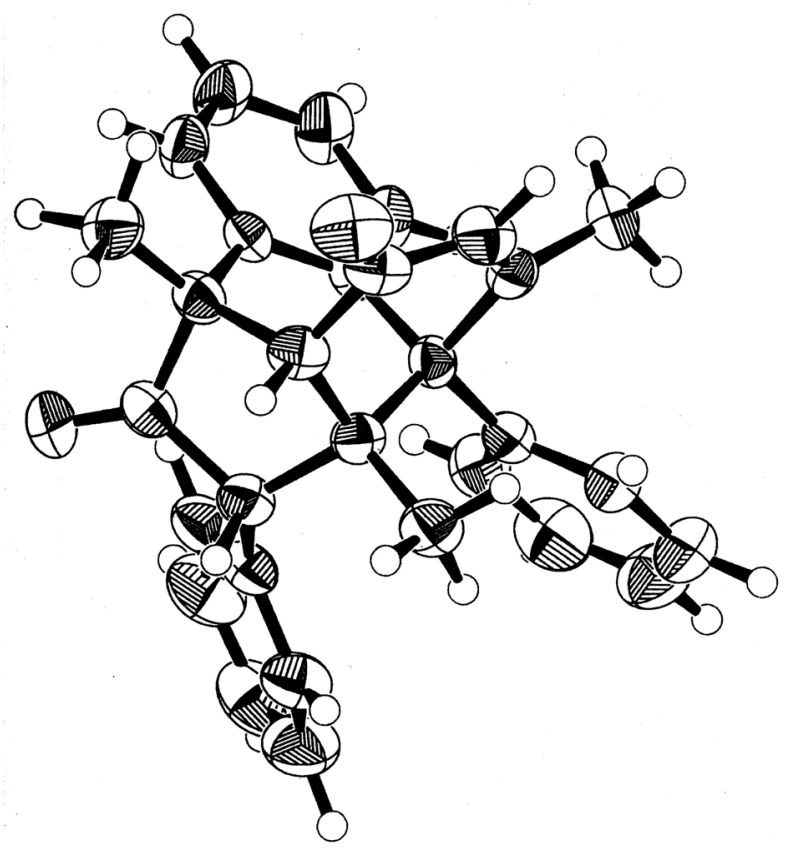

Figure S3. ORTEP drawing of $\mathbf{5 b}$. 


\section{Kinetic Simulation for $\mathrm{BF}_{3} \cdot \mathrm{Et}_{2} \mathrm{O}$ Catalyzed Reactions of 1 .}

The theoretical product ratio $\mathrm{P}_{\mathrm{A}} / \mathrm{P}_{\mathrm{B}}$ for each concentration of $\mathrm{BF}_{3} \bullet \mathrm{Et}_{2} \mathrm{O}$ was calculated by equation 4 . The estimated kinetic parameters were obtained by using Solver program in Microsoft Excel software, reducing the difference between calculated and experimental values of $\mathrm{P}_{\mathrm{A}} / \mathrm{P}_{\mathrm{B}}$ on the basis of the least squares method.

\section{References}

(S1) Kokubo, K.; Yamaguchi, H.; Kawamoto, T.; Oshima T. J. Am. Chem. Soc. 2002, 124, 8912-8921.

(S2) (a) Kokubo, K; Koizumi, T; Oshima, T. Tetrahedron Lett. 2001, 5025-5028. (b) Koizumi, T.; Mochizuki, E.; Kokubo, K.; Oshima T. J. Org. Chem. 2004, 69, 4577-4585. 